\title{
EFEITO DE DOSES DE INSETICIDA E ADJUVANTE NO CONTROLE DE PHYLLOCNISTIS CITRELLA STAINTON (LEPIDOPTERA: GRACILLARIIDAE) EM LIMA ÁCIDA TAHITI
}

\author{
Marcos Doniseti Michelotto', Willians César Carrega², Juliana Altafin Galli', Maycon Ferraz', Tamiris Marion \\ de Souza², Fernando Alves de Azevedo ${ }^{3}$
}

${ }^{1}$ Agência Paulista de Tecnologia dos Agronegócios (APTA), Polo Regional Centro Norte de Pindorama, SP. ${ }^{2}$ Universidade Estadual Paulista - UNESP, Câmpus de Jaboticabal, SP. ${ }^{3}$ Centro de Citricultura "Sylvio Moreira", Instituto Agronômico, Cordeirópolis, SP. E-mail: michelotto@apta.sp.gov.br

\begin{abstract}
RESUMO
A larva-minadora-dos-citros, Phyllocnistis citrella Stainton, 1856 (Lepidoptera, Gracillariidae), é uma das principais pragas da cultura da lima ácida Tahiti e seu principal método de controle é o químico. O objetivo deste trabalho foi avaliar o efeito de tratamentos inseticidas associados a adjuvante, no aumento da eficiência de controle da larva-minadora. Para isso, foi instalado um experimento em delineamento estatístico de blocos casualizados com cinco tratamentos e quatro repetições. Os tratamentos consistiram de uma testemunha sem aplicação de defensivos e o uso do inseticida Ampligo ${ }^{\circledR}$ (clorantraniliprole + lambda-cialotrina) na dose recomendada, na metade da dose recomendada e associado ao adjuvante. Foram realizadas três aplicações respeitando o período de carência do produto, avaliou-se os danos da larva-minadora em cinco ramos da planta central e a colheita dos frutos, determinando-se o número e a massa $(\mathrm{kg})$ de frutos por planta. Os dados foram submetidos à análise de variância pelo teste $\mathrm{F}$ e as médias foram comparadas pelo teste de Tukey $(P \leq 0,05)$. Também foram realizadas técnicas de análise multivariada (componentes principais e análise de agrupamento). Todos os tratamentos foram eficientes na diminuição dos sintomas de ataque da larva-minadora, com destaque a aplicação de inseticida em meia dosagem e a aplicação de inseticida em meia dosagem com o adjuvante. A análise multivariada foi eficiente em discriminar os tratamentos de aplicação de inseticidas e adjuvantes, e estabelecer uma relação direta entre a larva-minadora do citros e a diminuição na produção de frutos.

Palavras-chaves: Citrus latifolia Tanaka; controle químico; estatística multivariada; produção.
\end{abstract}

\section{EFFECT OF INSECTICIDE AND ADJUVANT DOSES IN CONTROL OF PHYLLOCNISTIS CITRELLA STAINTON (LEPIDOPTERA: GRACILLARIIDAE) IN ACID LIME TAHITI}

\begin{abstract}
Citrus leaf miner, Phyllocnistis citrella Stainton, 1856 (Lepidoptera, Gracillariidae), is one of the main pests of the culture of acid lime Tahiti and control chemical is the primary method of control. The objective of this study was to test the effect of insecticide treatments associated with adjuvant, in increasing the efficiency of control of citrus leaf miner in acid lime Tahiti. An experiment was installed in statistical design of randomized blocks with five treatments and four repetitions. The treatments consisted of a control treatment without application of pesticides and the use of the insecticide Ampligo $^{\circledR}$ (clorantraniliprole + lambda-cialotrina) at the recommended dosage, at half the recommended dose and associated with the adjuvant. Three applications were carried out respecting the shortage period of the product. The damages of citrus leaf miner were assessed in five branches of the central plant. Fruits were harvest and determining the number and the mass $(\mathrm{kg})$ of fruit per plant. The data were subjected to analysis of variance $(\mathrm{F}$ test) and the means were compared by Tukey test $(P \leq 0.05)$. Also the data were submitted to multivariate analysis using hierarchical cluster analysis techniques and principal component analysis (PCA). All treatments were effective in reducing the attack symptoms of citrus leaf miner, highlighting insecticide in half dosage and application of insecticide in half dosage with the adjuvant. The multivariate analysis was effective in discriminating application treatments of insecticides and adjuvant, and show a direct relationship between the citrus leaf miner and the decrease in fruit production.
\end{abstract}

Keywords: Citrus latifolia Tanaka; chemical control; multivariate statistics; production. 


\section{INTRODUÇÃO}

Segundo dados da Food and Agricultural Organization (FAO, 2018) a produção de lima ácida 'Tahiti' (Citrus latifolia Tanaka) no mercado global, vem se expandindo nos últimos anos. Em 2016, a produção mundial de limões e limas foi superior a 19,6 milhões de toneladas em uma área de cerca de 1,2 milhões de hectares. Entre os principais produtores destacaram-se Índia, México, China, Argentina e Brasil. Em conjunto, esses países foram responsáveis por $66 \%$ do total produzido.

O Brasil possui uma área de 47.279 hectares e produção estimada de 1,26 milhões de toneladas (FAO, 2018). A produção nacional de lima ácida 'Tahiti' concentra-se no estado de São Paulo (69\%), seguido de Minas Gerais (10\%) e Bahia (6\%) (AGRIANUAL, 2017).

A presença de insetos-pragas é considerada um dos principais fatores limitantes na produtividade e qualidade de frutas (KUMAR et al., 2017). Segundo Parra et al. (2003), plantas do gênero Citrus são afetadas por inúmeras pragas, que causam danos diretos e indiretos. Dentre as principais pragas, a Phyllocnistis citrella Stainton, 1856 (Lepidoptera, Gracillariidae) é considerada de grande importância na produção de citros e amplamente distribuída em todo o mundo (FLORES; VEITÍA, 2016; SARADA et al., 2014; PAIVA; YAMAMOTO, 2014). Vulgarmente chamada de larva-minadora-dos-citros, ela foi identificada pela primeira vez no Brasil em março de 1996, na região de Limeira, Estado de São Paulo (PRATES et al., 1996). Dois meses após, disseminou-se por todo o Estado (MONTES et al., 2001). As larvas de $P$. citrella atacam preferencialmente as folhas novas de citros, que apresentam minas típicas, com o tecido foliar assumindo uma coloração prateada e tornam-se retorcidas, ocorrendo morte do tecido foliar e queda prematura das folhas (KNAPP et al., 1995). De acordo com Foelkel et al. (2009) essa praga é uma ameaça à citricultura devido às perdas econômicas que pode causar. Segundo Sarada et al. (2014), as larvas causam cerca de $85 \%$ de danos nas folhas das plantas de citros.

Pela incidência dessa praga, o Citrus é altamente dependente dos agroquímicos para reduzir e diminuir os danos e prejuízos ocasionados pelos insetos e ácaros, e em determinados casos, para evitar a transmissão de fitopatógenos (YAMAMOTO et al., 2009).
Segundo Neves et al. (2010) o uso de inseticidas em cítricos, principalmente no Estado de São Paulo, aumentou seis vezes. Para Costa et al. (2003) a adição de substâncias químicas que permitam a solubilização e dispersão do ingrediente ativo para o aumento da deposição, adesão, molhamento e retenção da calda pulverizada podem também aumentar a toxicidade dos inseticidas sobre o alvo.

Para que um ingrediente ativo possa ser mais eficientemente aproveitado em uma pulverização, considerando as chuvas, deve-se melhorar a interação das gotas em contato com o alvo, através do uso de adjuvantes capazes de reduzir a tensão superficial do líquido, promovendo maior efeito molhante, espalhante e penetrante (GREEN; HAZEN, 1998).

Acosta et al. (2015) salientam que o uso irracional de inseticidas pode trazer consequências como o desequilíbrio ecológico, resistência a inseticidas, surgimento de pragas secundárias e diminuição dos inimigos naturais. Diante disso, o objetivo deste trabalho foi avaliar o efeito de doses do inseticida Clorantraniliprole + lambda-cialotrina chlora (Ampligo ${ }^{\circledR}$ ) e do adjuvante Disperse Ultra ${ }^{\circledR}$ no controle de $P$. citrella em lima ácida Tahiti, visando buscar um manejo mais econômico e sustentável.

\section{MATERIAL E MÉTODOS}

O experimento foi instalado em área experimental da Apta, Polo Regional Centro Norte, no município de Pindorama, SP (coordenadas geográficas: S $21^{\circ} 13^{\prime} 16,6^{\prime \prime}$ e W $48^{\circ}$ $\left.54^{\prime} 21,5^{\prime \prime}\right)$, altitude $520 \mathrm{~m}$, em pomar de lima ácida, clone nucelar de Tahiti, denominado IAC-5 enxertado em trifoliata Flying Dragon, implantado em março de 2013 com altura das plantas de 2,0 m aproximadamente, espaçamento 7,0 $\times 4,0 \mathrm{~m}$, estando na fase de formação e intensa brotação. Foram realizadas duas adubações de cobertura em 01/09 e 01/11/2016 da formula 20-5-20 de NPK na dosagem de $400 \mathrm{~g}$ por planta e capinas manuais e mecânicas sempre que necessárias.

O delineamento estatístico utilizado foi o de blocos casualizados com cinco tratamentos e quatro repetições. Os tratamentos, ilustrados na Tabela 1, consistiram de uma testemunha sem aplicação de defensivos e o uso do inseticida isoladamente ou em associação com o adjuvante Disperse Ultra (121 g L ${ }^{-1}$ de Nitrogênio solúvel em 
água; $44 \mathrm{~g} \mathrm{~L}^{-1}$ de $\mathrm{P}_{2} \mathrm{O}_{5}$; agente alcalinizante, estabilizante, tensoativos/surfactante), um adjuvante a base de moléculas orgânicas que promovem a translocação de elementos de baixa mobilidade, assim como outros componentes da calda de pulverização. 0 inseticida Ampligo ${ }^{\circledR}$ (lambda-cialorina + chlorantranilipole) é de contato e ingestão, recomendado para psilídeo
[Diaphorina citri Kuwayama (Hemiptera: Psyllidae)], mosca-negra-dos-citros [Aleurocanthus woglumi Ashby, 1915 (Hemiptera: Aleyrodidae)] e larva-minadora.

Cada parcela foi constituída de três plantas, abrangendo área de $84 \mathrm{~m}^{2}$ por parcela, sendo as plantas dispostas em linha única.

Tabela 1. Tratamentos e doses empregadas nos experimentos.

\begin{tabular}{lcc}
\hline Tratamentos & $\begin{array}{c}\text { Dosagem Inseticida } \\
(\mathrm{mL} / 100 \mathrm{~L} \text { de água })\end{array}$ & $\begin{array}{c}\text { Dosagem Adjuvante } \\
(\mathrm{mL} / 100 \mathrm{~L} \text { de água })\end{array}$ \\
\hline 1. Testemunha & - & - \\
2. ${ }^{1} \mathrm{DR}$ & 10,0 & - \\
3. $1 / 2 \mathrm{DR}$ & 5,0 & - \\
$4 .{ }^{1} \mathrm{DR}+\mathrm{A}$ & 10,0 & 10,0 \\
$5.1 / 2 \mathrm{DR}+\mathrm{A}$ & 5,0 & 30,0 \\
\hline
\end{tabular}

${ }^{*}$ Inseticida Ampligo ${ }^{\oplus}$ do grupo dos piretróides (i.a.: lambda-cialotrina) e antranilamida (i.a.: chlorantranilipole) na dosagem mínima recomendada (DR); ${ }^{1}$ DR ( $100 \%$ da dose); $1 / 2 \mathrm{DR}$ (50\% da dose). Adjuvante (A), Disperse Ultra.

As aplicações dos produtos foram realizadas com pulverizador costal elétrico (Pulmic Energy), munido de um bico Conet Jet TXVK-8 e pressão de trabalho de 28 psi. A vazão foi definida de 1 litro de calda por planta e sua padronização foi utilizada em todos os tratamentos de forma a promover o molhamento de toda a copa.

Foram realizadas três aplicações do produto comercial Ampligo ${ }^{\circledR}$ (clorantraniliprole + lambda-cialotrina) nas datas: 24/10, 11/11 e 30/12/2016, sendo respeitado o período de carência do produto. Foram realizadas 7 avaliações semanais, iniciando no dia 21/10/16 (avaliação previa) e nos dias 31/10/16 (7 DA1aA), 07/11/16 (14 DA1aA), 21/11/16 (10 DA2A), 28/11/16 (17 DA2a), 02/01/17 (3DA3A) e 10/01/17 (11DA3aA).

Em cada parcela foram avaliados cinco ramos da planta central, cortados com o auxílio de uma tesoura e acondicionados em sacos plásticos devidamente identificados e conduzidos ao laboratório, onde foi realizada a contagem do número de folhas com sintomas recentes de ataque da larva-minadora por brotos, utilizando um microscópio estereoscópio com aumento de 20 vezes.

Foi realizada a colheita de frutos no estádio de maturação $\mathrm{C} 2$ de todas as plantas da parcela em diferentes datas, 21/11/16, 07/11/16, 28/11/16, 23/01/17 e 24/02/17, em função da característica da espécie que possui diversas frutificações e do período de desenvolvimento do fruto que necessita até 170 dias dependendo do regime hídrico e térmico de cada região (DONADIO et al., 2005). Para isso, realizou-se a colheita manual de todos os frutos da planta central de cada parcela, determinando o número de frutos e massa $(\mathrm{kg})$ de frutos por planta. Para fins de comparação entre os tratamentos foram computados o total produzido de frutos e a massa total de frutos no período. A produção foi mensurada 90 dias após o início do experimento, em função do período de desenvolvimento dos frutos

Os dados obtidos foram submetidos à análise de variância pelo teste $\mathrm{F}$ e as médias foram comparadas pelo teste de Tukey $(P \leq 0,05)$, sendo os dados de porcentagem de infestação transformados em $\operatorname{arcsen}(x / 100)^{-1}$. A eficiência dos tratamentos na redução da larva-minadora foi calculada pela fórmula proposta por Abbott (1925).

Também foram realizadas técnicas de análise multivariada, utilizando a análise de componentes principais, e análise de agrupamento. As análises foram processadas no programa Statistica versão 7.0 (STATSOFT, 2010).

A análise de agrupamento hierárquica foi processada utilizando-se como coeficiente de semelhança a distância euclidiana e o método Ward, e a análise de agrupamento não hierárquica utilizou-se do método K-means, sendo $\mathrm{K}$ o número de grupos indicados na análise de agrupamento hierárquica. 


\section{RESULTADOS E DISCUSSÃO}

$\mathrm{Na}$ avaliação prévia, realizada antes da aplicação dos produtos, observou-se que não houve diferença significativa para os parâmetros avaliados, demonstrando que a área estava uniforme e sem influência de inseticidas, portanto apta para a aplicação dos tratamentos (Tabela 2).

$\mathrm{Na}$ avaliação realizada aos sete dias após a primeira aplicação (7 DA1 $\mathrm{A} A)$ observou-se diferença significativa entre a testemunha e os demais tratamentos para o percentual de folhas com lesão, na qual o inseticida nas duas dosagens (50\% e $100 \%$ da DR) com ou sem adição do adjuvante apresentaram eficiência de controle variando de 59,5\% a 64,5\% (Tabela 2). Aos 14 DA1aA, foi observado comportamento similar aos 7 DA1 a. Contudo, os tratamentos sem adjuvantes, independente da dose, apresentaram os menores danos da larva-minadora, com eficiência de controle de $82,5 \%$ ( $100 \%$ da DR) e $73,6 \%$ (50\% da DR) (Tabela 2). Nas avaliações a partir dos 10 DA2A não foram constatadas diferenças estatísticas entre os tratamentos analisados, apesar dos dados mostrarem uma tendência de maior infestação na testemunha em relação aos demais tratamentos nas quatro últimas épocas de avaliação.

Tabela 2. Porcentagem de folhas com lesões da larva-minadora ( $L$ ) e eficiência de controle (Ec, \%) nos diferentes tratamentos. Pindorama, SP.

\begin{tabular}{|c|c|c|c|c|c|c|c|c|c|c|c|c|c|}
\hline \multirow{2}{*}{ Trat. } & \multirow{2}{*}{$\begin{array}{c}\text { Prévia } \\
\mathrm{L}\end{array}$} & \multicolumn{2}{|c|}{7 DA1ㅇA } & \multicolumn{2}{|c|}{14 DA1ㅇA } & \multicolumn{2}{|c|}{$10 \mathrm{DA} 22^{\circ} \mathrm{A}$} & \multicolumn{2}{|c|}{$17 \mathrm{DA} 2^{\circ} \mathrm{A}$} & \multicolumn{2}{|c|}{3 DA3A } & \multicolumn{2}{|c|}{11 DA3A } \\
\hline & & $\mathrm{L}$ & Ec & $\mathrm{L}$ & Ec & $\mathrm{L}$ & Ec & $\mathrm{L}$ & Ec & $\mathrm{L}$ & Ec & $\mathrm{L}$ & Ec \\
\hline Test. & 76,6 & $60,3 \mathrm{a}$ & - & $41,6 \mathrm{a}$ & - & 30,8 & - & 37,0 & - & 72,3 & - & 46,3 & - \\
\hline${ }^{1} \mathrm{DR}$ & 82,0 & $24,4 \quad b$ & 59,5 & $7,3 \mathrm{~b}$ & 82,5 & 11,1 & 64,0 & 6,8 & 81,6 & 43,8 & 39,4 & 34,0 & 26,6 \\
\hline $1 / 2 \mathrm{Dr}$ & 80,7 & $22,8 \quad b$ & 62,2 & $11,0 \mathrm{~b}$ & 73,6 & 14,6 & 52,6 & 18,1 & 51,1 & 55,9 & 22,7 & 30,3 & 34,6 \\
\hline${ }^{1} \mathrm{DR}+\mathrm{A}$ & 78,4 & $22,0 \quad b$ & 63,5 & $14,8 \mathrm{ab}$ & 64,4 & 15,5 & 49,7 & 21,4 & 42,2 & 43,5 & 39,8 & 37,0 & 20,1 \\
\hline $1 / 2 \mathrm{Dr}+\mathrm{A}$ & 69,6 & $21,4 \quad b$ & 64,5 & $17,0 a b$ & 59,1 & 16,5 & 46,4 & 19,9 & 46,2 & 42,4 & 41,4 & 25,8 & 44,3 \\
\hline Teste $\mathrm{F}$ & $0,33^{\text {ns }}$ & $8,38^{* *}$ & & $4,66^{*}$ & & $1,29^{\text {ns }}$ & & $2,18^{\text {ns }}$ & & $2,21^{\text {ns }}$ & & $0,63^{\text {ns }}$ & \\
\hline CV (\%) & 23,24 & 21,87 & & 37,26 & & 46,59 & & 49,97 & & 23,63 & & 37,41 & \\
\hline
\end{tabular}

Médias seguidas de mesma letra na coluna não diferem pelo teste de Tukey a $5 \%$ de probabilidade de erro; ${ }^{\text {ns }}=$ não significativo; ${ }^{*}$ significativo a $5 \%$; ${ }^{* *}$ significativo a $1 \% .{ }^{1} \mathrm{dr}(100 \%$ da dose); $1 / 2 \mathrm{dr}(50 \%$ da dose). Adjuvante (A), Disperse Ultra.

Para o número de frutos/planta, observou-se que o maior número de frutos foi obtido com $100 \%$ da DR, com aumento de $55 \%$ em relação à testemunha (Figura 1). Contudo, o tratamento com $100 \%$ da DR, não diferiu da meia dosagem com e sem adjuvante. Em relação à testemunha, 50\% da DR apresentou aumento de $39,1 \%$ com adição do adjuvante e $51,7 \%$, sem adjuvante. Para a produção de frutos em $\mathrm{kg} /$ planta, observou-se comportamento similar ao obtido para o número de frutos/planta, ou seja, o tratamento com $100 \%$ da DR foi o mais produtivo, com $53,7 \%$ de aumento em relação à testemunha, porém sem diferir dos demais tratamentos, exceto a testemunha.

Figura 1. Número de frutos por planta (a) e peso médio de frutos por planta (b) produzidos durante o período de avaliação (dezembro/2016 a abril de 2017) do experimento. Pindorama, SP. Barras seguidas por letras diferentes diferem significativamente, para cada tratamento, pelo teste de Tukey a 5\%. (a) $\mathrm{F}=7,99 * *$; $\mathrm{CV}=12,82$; (b) $6,05^{* *}$; $\mathrm{CV}=14,03 \%$. 


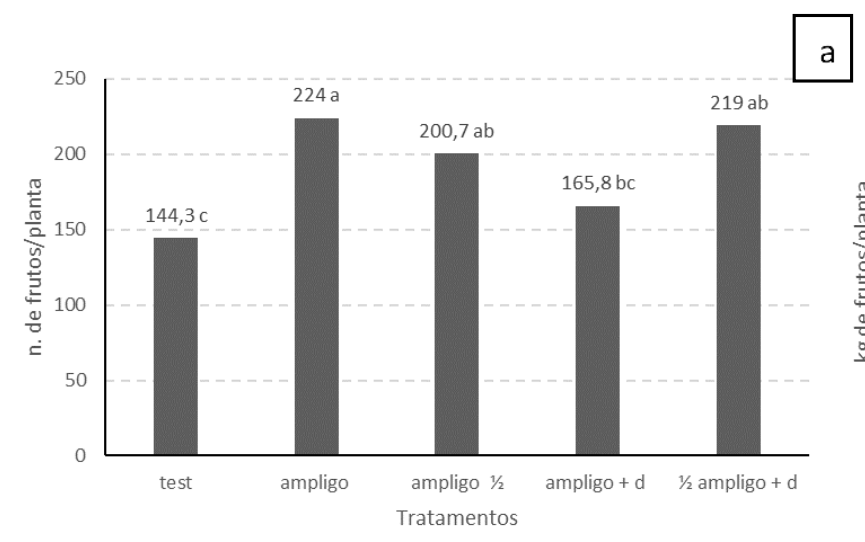

Os resultados da análise de agrupamento hierárquico estão representados na Figura 2 . Considerando no dendograma um ponto de quebra na distância de 2,5 , se identificaram três grupos. O primeiro representado pelo tratamento 4 (100\% da DR + adjuvante), o segundo,

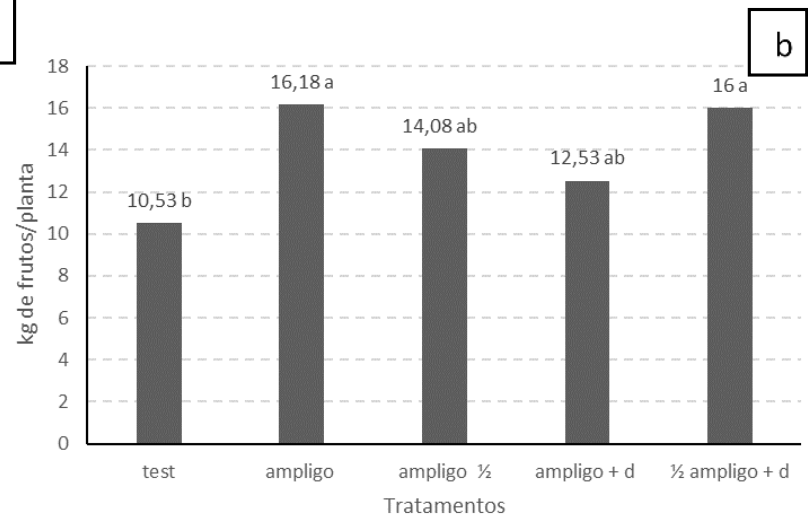

representado pelos tratamentos 2 , 3 e $5(100 \%$ da DR, $50 \%$ da DR e $50 \%$ da DR + adjuvante, respectivamente), e o terceiro grupo, representado pela Testemunha (T1).

Figura 2. Classificação dos tratamentos de aplicação de doses de inseticida com ou sem adjuvante, pela análise de agrupamento hierárquica pelo método Ward.

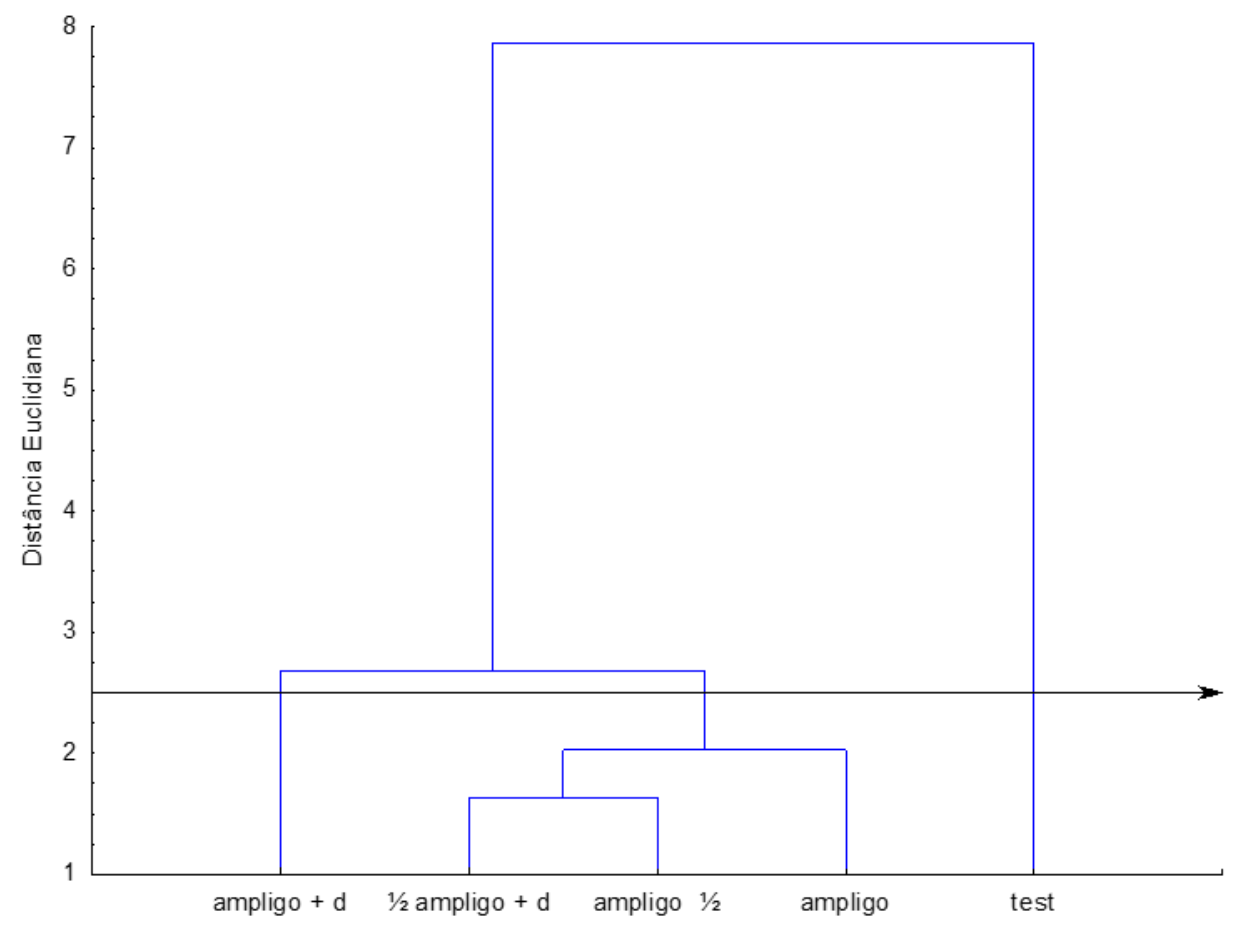

O resultado da análise de agrupamento não hierárquica está descrito na Figura 3. Considerando o agrupamento de médias (Kmeans), sendo $K=3$, verificou-se que a Testemunha (Grupo 2) apresentou as maiores porcentagens de danos por larva-minadora em todas as épocas de avaliação e, consequentemente, as menores produções de frutos/planta e $\mathrm{kg} /$ planta. $\mathrm{O}$ grupo 3 , formado pelos tratamentos 2 , 3 e 5 , mostrou-se superior ao tratamento 4 ( $100 \%$ da DR + adjuvante), por ser o grupo com menor porcentagem de folhas com sintomas e maior produção. A superioridade do grupo 3 na produção também foi observada na análise de variância (Figura 1). 
Figura 3. Análise de agrupamento não hierárquica contendo a ordenação dos grupos com relação a porcentagem de folhas com sintomas de larva-minadora nas diferentes épocas de avaliação e produção de frutos.

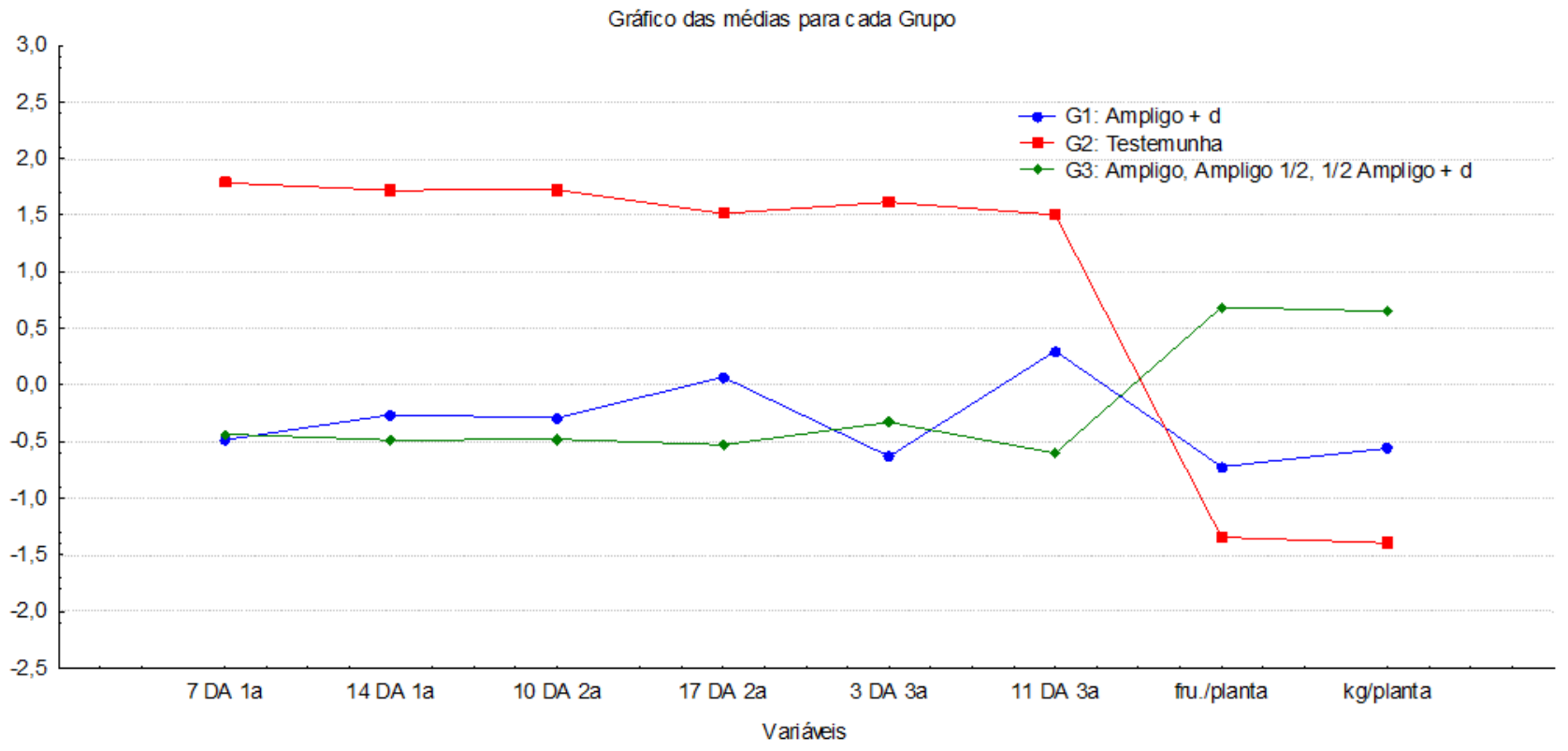

A análise dos componentes principais foi realizada para obtenção de uma visão geral dos tratamentos. Nela, $92,64 \%$ da variação presente nos dados originais foram explicadas pelos dois primeiros componentes principais (CP1 e CP2) (Tabela 3). O primeiro CP explicou $85,43 \%$ da variância total e o segundo, $7,20 \%$. Segundo Teixeira et al. (2012), é importante frisar que o primeiro componente principal sempre contém o maior número de informação dos valores originais, sendo o que representa os resultados mais relevantes.

Todas as características apresentaram poder discriminatório no $\mathrm{CP} 1$ (valores próximos a
1) (Tabela 3). Como todas as épocas de avaliação possuem sinal positivo, indicam que unidades mais à direita no gráfico sofrem maior influência dessas características. Já as correlações entre as variáveis fruto/planta e $\mathrm{kg} /$ planta e o componente principal 1 são negativas, indicando que unidades a esquerda sofrem influência dessas características. No CP2 todas as características apresentaram correlações baixas (menores que 0,5), portanto não serão consideradas para fins de discussão.

Tabela 3. Coeficiente de correlação das variáveis

\begin{tabular}{lrrrrr}
\hline & Variável & CP 1 & CP 2 & CP 3 & CP 4 \\
\hline 7 DA 1a & & $0,940843^{*}$ & 0,222170 & 0,240827 & $-0,086353$ \\
14 DA 1a & $0,955052^{*}$ & 0,232257 & $-0,092582$ & $-0,159252$ \\
\hline
\end{tabular}




\begin{tabular}{lllll}
\hline 10 DA 2a & $0,965242^{*}$ & 0,230233 & $-0,093783$ & $-0,080660$ \\
17 DA 2a & $0,932562^{*}$ & 0,086595 & $-0,350385$ & 0,007757 \\
3 DA 3ㅁ & $0,891917^{*}$ & 0,225182 & 0,190202 & 0,342928 \\
11 DA 3a & $0,870088^{*}$ & $-0,296424$ & 0,367948 & $-0,140338$ \\
Fruto/planta & $-0,907583^{*}$ & 0,396717 & 0,137301 & $-0,007562$ \\
Kg/planta & $-0,927245^{*}$ & 0,341556 & 0,092813 & $-0,122234$ \\
\hline Variância exploratória & 6,834733 & 0,576428 & 0,397162 & 0,191677 \\
\hline Proporção da variância Total (\%) & 85,43416 & 7,20535 & 4,96452 & 2,39597 \\
\hline
\end{tabular}

Obs.: *variáveis com importância significativa para o respectivo fator. Sinais iguais indicam relação direta, e contrário, indireta.

Na Figura 4 encontra-se a dispersão bidimensional dos escores dos componentes principais 1 e 2 e os grupos de tratamentos. Verifica-se a divergência de todos os tratamentos com relação a testemunha. Neste gráfico, ficou evidente que a testemunha está fortemente associada as maiores porcentagens de folhas com sintomas de ataque da larva-minadora, em todas as épocas de avaliação. Já os tratamentos do grupo 3 são os mais divergentes da testemunha e fortemente guiados pelas características de produção, confirmando a superioridade desses tratamentos obtida na análise de agrupamento.

Figura 4. Dispersão bidimensional dos escores dos componentes principais 1 e 2 e dos tratamentos de aplicação de inseticidas na porcentagem de folhas com lesões da larva-minadora e produção de frutos.

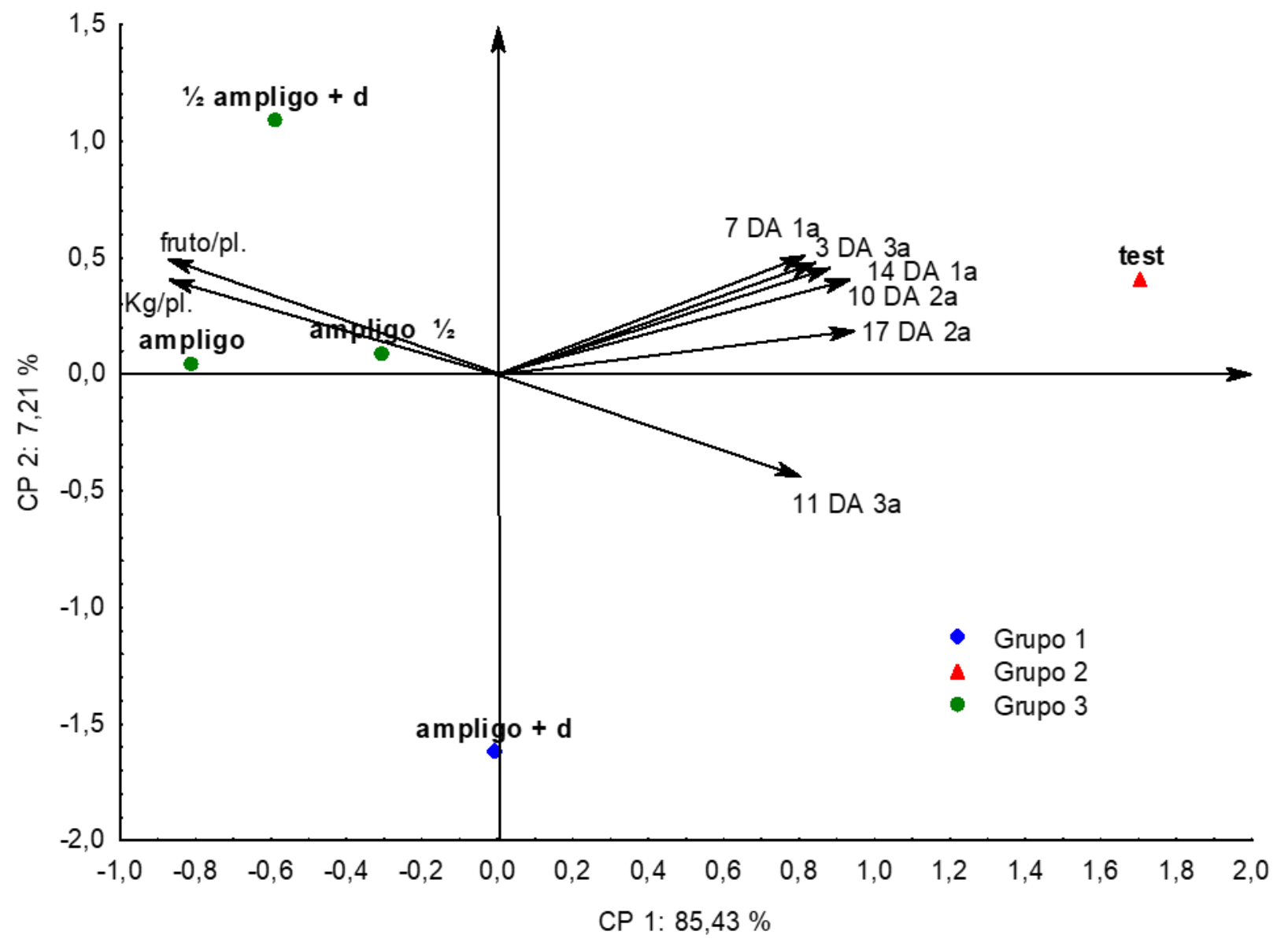

Os resultados observados no presente estudo indicam que os danos causados pela presença de $P$. citrella ao longo do experimento foram de $48 \%$. Nossos resultados, coincidem com pesquisas realizadas por Diez et al. (2006) que verificaram danos médios de $51 \%$ causados por essa mesma praga. Contudo, salientamos que os danos desse inseto podem ser maiores, pelas 
perdas indiretas decorrentes da presença de fitopatógenos oportunistas.

No presente estudo, analisando apenas a influência de $P$. citrella, verificamos que os danos causados pela larva-minadora podem comprometer o potencial produtivo da lima ácida Tahiti, causando reduções de até $35 \%$. Resultado semelhante foi constatado por Hunsberger et al. (1996) trabalhando com Tahiti no sul da Flórida, no qual verificaram redução de $37,7 \%$ no rendimento do limão devido ao dano de $P$. citrella. Essa redução está atrelada ao fato da larva-minadora afetar diretamente a atividade fotossintética. Ao se alimentarem das folhas, as larvas afetam negativamente a passagem de luz, impedindo a fase bioquímica da fotossíntese. Arshad et al. (2018) constataram que à medida que o dano dessa praga aumenta, a taxa fotossintética e a eficiência no uso da água foram significativamente afetadas. Além disso, as perdas indiretas decorrentes da presença de patógenos como a bactéria fitopatogênica gramnegativa Xanthomonas citri subsp. citri (Xac), agente etiológico do cancro cítrico pode aumentar ainda mais os danos e causar reduções ainda mais drásticas à cultura. Segundo Sarada et al. (2014) as perdas indiretas causadas pela larvaminadora podem favorecer o aumento da doença do câncer cítrico em até $50 \%$.

Algumas pesquisas têm indicado que estratégias como o uso do controle biológico tem sido uma alternativa positiva para reduzir os níveis de infestação dessa praga. Contudo, o controle biológico ainda deve ser utilizado dentro do manejo integrado de pragas, ou seja, não excluindo totalmente $\mathrm{o}$ uso de inseticidas. Montes et al. (2001), verificaram que a ocorrência de parasitoides nativos apresentou uma taxa de parasitismo em torno de $35 \%$, mostrando que os inimigos naturais nativos auxiliam no controle da larva-minadora, porém necessitando de medidas complementares. Entre elas, o uso de inseticidas, como o Ampligo $^{\circledR}$ é uma opção viável.

Constatou-se que o uso de $50 \%$ e $100 \%$ da dose recomendada deste produto, promoveram resultado similar, deixando evidente que reduzir a dose é uma opção viável para controlar ou minimizar os impactos da ocorrência de $P$. citrella em lima ácida Tahiti. $O$ uso da meia dosagem pode promover uma economia de $50 \%$ nos custos com inseticidas para o controle desse inseto-praga. A combinação do chlorantraniliprole com lambda-cyhalothrin
(Ampligo ${ }^{\circledR}$ ) atua paralisando os músculos dos leptópteros, causando interrupção na alimentação e, consequentemente, levando a morte dos insetos sensíveis (SYNGENTA, 2017). A eficiência desse produto em insetos-praga tem sido comprovada por outros autores. Cessa et al. (2013) constatou mortalidade superior a $80 \%$ de lagartas de Spodoptera frugiperda (Smith, 1797) (Lepidoptera: Noctuidae) alimentadas com folhas de milho e de feijão imersas em soluções com o inseticida Ampligo ${ }^{\circledR}$. Sen et al. (2017) testando a mistura do chlorantraniliprole + lambdacyhalothrin observaram cerca de $90 \%$ de redução na infestação de Leucinodes orbonalis Guenée, 1854 (Lepidoptera: Pyralidae) e aumento do rendimento em berinjela. Em algodão, Bajya et al. (2015), observaram que as menores doses do Ampligo $150 \mathrm{ZC}$ foi o melhor tratamento e com as maiores produtividades.

As informações evidenciadas no presente estudo, mostraram que todos os tratamentos foram eficientes na diminuição dos sintomas de ataque de $P$. citrella. Entretanto, levando-se em conta o impacto ambiental e econômico, merece destaque a aplicação de inseticida em meia dosagem com adição do adjuvante, por ter efeito no combate dessa praga e manter os níveis ideais de produção dos frutos de lima ácida Tahiti. Além disso, a análise multivariada foi eficiente em discriminar os tratamentos de aplicação de inseticidas isolados e em mistura com adjuvantes, e estabelecer uma relação direta entre a larvaminadora do citros e a diminuição na produção de frutos.

\section{REFERÊNCIAS}

ABBOTT, W. S. A method of computing the effectiveness of insecticide. Journal Economic Entomology, v.18, n.1, p.265-267, 1925. https://doi.org/10.1093/jee/18.2.265a

ACOSTA, F.A.G.; CAMPOS, J. C.; VIRGEN, M. O. E.; BERMUDEZ, A.R.; SANDOVAL, G. R. P.; BLANCO, J. M.C. Parasitismo del minador de la hoja de los cítricos (Phyllocnistis citrella Stainton) en limón persa en Xalisco, Nayarit. Entomología Mexicana, v. 2, p. 186-192, 2015. file:///C:/Users/Usuario/Downloads/Parasitismo_ del_minador_de_la_hoja_de_lo.pdf

AGRIANUAL: Anuário da agricultura brasileira. 2017. Citros. São Paulo: FNP. 482p. 
ARSHAD, M.; ULLAH, M. I.; AFZAL, M.; AATIF, H. M.; IFTIKHAR, Y.; MOLINA-OCHOA, J.; FOSTER, J. E. Association of citrus leafminer Phyllocnistis citrella (Lepidoptera: Gracillariidae) damage with physiological parameters and larval weight in Citrus reticulata. International Journal of Tropical Insect Science, v. 38, n. 1, p. 26-32, 2018. https://doi.org/10.1017/S1742758417000273

BAJYA, D. R.; BAHETI, H. S.; RAZA, S. K. Field efficacy of newer insecticide formulation Ampligo 150 ZC against bollworm complex in cotton. Journal of Cotton Research and Development, v. 29, n. 1, p. 94-98, 2015. http://www.crdaindia.com/fileserve.php?FID=14 7

CESSA, R. M. A.; MELO, E. P. de; LIMA JUNIOR, I. D. S de. Mortalidade de Spodoptera frugiperda (JE Smith) (Lepdoptera: noctuidae) alimentadas com folhas de milho e feijoeiro imersas em soluções contendo inseticidas. Revista Agrogeoambiental, v. 5, n. 1, 2013. https://doi.org/10.18406/2316-

$\underline{1817 v 5 n 12013434}$

COSTA, E. A. D.; ALMEIDA, J. E. M.; LOUREIRO, E. S.; SANO, A. H. Compatibilidade de adjuvantes no desenvolvimento "in vitro" dos fungos entomopatogênicos Metarhizium anisopliae (Metsch.) Sorokin e Beauveria bassiana (Bals.) Vuillemin. STAB: Açúcar, Álcool e Subprodutos, v.22, n.2, p.38-41. 2003.

DIEZ, P. A.; PEÑA, J. E.; FIDALGO, P. Population dynamics of Phyllocnistis citrella (Lepidoptera: Gracillariidae) and its parasitoids in Tafí Viejo, Tucumán, Argentina. Florida Entomologist, v. 89, n. 3, p. 328-335, 2006.

http://journals.fcla.edu/flaent/article/view/7555 2/73210. $\quad$ https://doi.org/10.1653/00154040(2006)89[328:PDOPCL]2.0.CO;2

DONADIO, L. C.; MOURÃO FILHO, F. A. A.; MOREIRA, C. S. Centros de origem, distribuição geográfica das plantas cítricas e histórico da citricultura no Brasil. In: MATTOS JÚNIOR, D.; DE NEGRI, J.D.; PIO, R.M.; POMPEU JÚNIOR, J. (Ed.). Citros. Campinas: Instituto Agronômico e Fundag, 2005. p.1-18.

FAO - Food and Agriculture Organization, 2018. Disponível em: <http://www.fao.org>. Acesso em: 10 mar. 2018.
FLORES, D. D. C.; VEITÍA, M. Evaluación de cinco insecticidas para el control de las poblaciones de Phyllocnistis citrella (Lepidoptera: Gracillariidae) en cultivos de toronja en Cuba. Revista Cubana de Ciencias Biológicas, v. 4, n. 3, p. 43-49, 2016. http://www.rccb.uh.cu/index.php/RCCB/article/v iew/117/228

FOELKEL, E.; REDAELLI, L. R.; JAHNKE, S. M.; LOSEKANN, P. B. Predation and parasitism of Cirrospilus neotropicus (Hymenoptera: Eulophidae) on Phyllocnistis citrella (Lepidoptera: Gracillariidae) in laboratory. Revista Colombiana de Entomología, v. 35, n. 2, p. 156-162, 2009. http://www.scielo.org.co/pdf/rcen/v35n2/v35n2 a08.pdf

GREEN, J. M.; HAZEN, J. L. Understanding and using adjuvants properties to enhance pesticide activity. In: INTERNATIONAL SYMPOSIUM ON ADJUVANTS FOR AGROCHEMISTS, 5., 1998, Tennessee. Proceedings... Memphis: ISAA, p. 2536.

HUNSBERGER, A. G. B.; PEÑA, J. E.; SCHAFFER, B. Relationships of citrus leafminer density to citrus damage and yield. In: INTERNATIONAL CONFERENCE MANAGING CITRUS LEAFMINER, 1996, Gainesville. Proceedings... Orlando: University of Florida, p. 86.

KNAPP, J. L.; ALBRIGO, L. G.; BROWING, H. W.; BULLOCK, R. C.; HEPPNER, J. B.; HALL, D. G.; HOY, M. A.; NGUYEN, R.; PEÑA, J. E.; STANSLY, P. A. Citrus leafminer, Phyllocnistis citrella Station: Current Status in Flórida. Gainesville: Flórida Cooperative Extensive Service, 1995. 35p.

KUMAR, K.; ARORA, P. K.; GILL, M. I. S. Breeding Avenues in Fruit Crops for Imparting Resistance Against Insect Pests. In: Breeding Insect Resistant Crops for Sustainable Agriculture. Singapore: Springer, 2017.2 p.289-322. https://doi.org/10.1007/978-981-10-6056-4 10

MONTES, S.M.N.M.; BOLIANI, A.C.; PAPA, G.; CERÁVOLO, L.C.; ROSSI, A.C.; NAMEKATA, T. Ocorrência de parasitóides da larva minadora dos citros, Phyllocnistis citrella Station, no município de Presidente Prudente, SP. Arquivos do Instituto Biológico, v.68, n.2, p.63-66, 2001. 
Disponível

e: <http://www.biologico.sp.gov.br/uploads/docs/a rq/V68_2/montes.pdf>

NEVES, M. F.; TROMBIN, V. G.; MILAN, P.; LOPES, F. F.; PEREIRA, F. C.; KALAKI, R. B. O retrato da citricultura brasileira. Ribeirão Preto: Markestrat, 2010.

PARRA, J. R. P.; OLIVEIRA, H. N.; PINTO, S. N. Guia ilustrado de pragas $\mathrm{e}$ insetos benéficos dos citros. Piracicaba: A. S. PINTO, 2003.140p.

PAIVA, P. E. B.; YAMAMOTO, P. T. Citrus caterpillars, with an emphasis on Helicoverpa armigera: a brief review. Citrus Research and Technology, v. 35, n. 1, p. 11-17, 2014. https://doi.org/10.5935/2236-3122.20140002

PRATES, H. S.; NAKANO, O.; GRANENA, S. "Minadora das folhas de citros" Phyllocnistis citrella, Stainton, 1856. Campinas: CATI, 1996. 129p. (Comunicado Técnico)

SARADA, G.; GOPAL, K.; GOURI SANKAR, T., MUKUNDA LAKSHMI, L., GOPI, V., NAGALAKSHMI, T., RAMANA, K. T. V. Citrus Leaf Miner (Phyllocnistis citrella Stainton, Lepidptera: Gracillariidae): Biolology and Management: A Review. Research and Reviews: Journal of Agriculture and Allied Sciences, v.3, n.3, p.39-48, 2014.

http://www.rroij.com/open-access/citrus-leafminer-phyllocnistis-citrella-stainton-lepidpteragracillariidae-biolology-and-management-areview-39-48.pdf

SEN, K.; SAMANTA, A.; ALAM, SK F.; DHAR, P. P.; SAMANTA, A. Field evaluation of a new ready mix formulation Ampligo 150 zc (chlorantraniliprole $9.3 \%$ + lambda cyhalothrin $4.6 \% \mathrm{zc}$ ) against shoot and fruit borer (Leucinodes orbonalis Guen.) infestation in Brinjal. Journal of Pharmacognosy and Phytochemistry, v.6, n.5, p.1674-1678, 2017. http://www.phytojournal.com/archives/2017/vol 6issue5/PartY/6-5-161-503.pdf

STATSOFT. Statistica (data analysis software system). Version 10. 2010. Disponível em: <http://www.statsoft.com>. Acesso em: 10 mar. 2018.

SYNGENTA. Relatório Técnico. Lisboa: Syngenta Crop Protection, 2017.
TEIXEIRA, G. H. A.; DURIGAN, J. F.; FERRAUDO, A. S.; ALVES, R. E.; O'HARE, T.J. Multivariate analysis of fresh-cut carambola slices stored under diferente temperatures. Postharvest Biology and Technology, v.63, n.1, p.91-97, 2012.

https://doi.org/10.1016/j.postharvbio.2011.09.00 $\underline{5}$

YAMAMOTO, P. T.; FELLIPE, M. R.; SANCHES, A. L.; COELHO, J. H.; GARBIM, L. F.; XIMENES, N. L. Eficácia de inseticidas para o manejo de Diaphorina citri Kuwayama (Hemiptera: Psyllidae) em citros. BioAssay, v.4, 2009.

Recebido para publicação em 21/03/2018

Revisado em 03/07/2018

Aceito em 13/08/2018 